\title{
Business and Management communication Cases: Challenges and Opportunities
}

\section{Priscilla S. Rogers}

University of Michigan, Ann Arbor

\section{Jone Rymer}

Wayne State University, Detroit, Michigan

$\mathrm{T}$

HIS SPECIAL ISSUE of Business Communication Quarterly publishes in a single volume the Casebook Project sponsored by the Association for Business Communication. Fifteen cases, all produced originally for this collection, were selected through a double-blind refereeing by a national Case Editorial Review Board of instructors highly experienced in the case method of teaching communications. The objective of the Casebook Project was to create a diverse set of cases relevant to business communication in the workplace and functional for a wide range of users in both undergraduate and MBA programs. Instructors may photocopy the cases freely for educational use (see the Permission to Photocopy section on the inside of the BCQ front cover). Issues to facilitate incorporating cases into teaching are discussed in this introduction, and further assistance in integrating the cases into curricula and classrooms is available at the $B C Q$ Web site, which includes teaching notes for most of the cases, as well as student sample communications:

\section{http://unix.cc.wmich.edu/rea/bcq}

The cases selected for this casebook were chosen from a large number submitted in response to an international call for cases by the

Acknowledgments: This casebook owes much to many, especially Linda P. Driskill, Rice University, and also Nina Kataja, International BBA Program, The Helsinki School of Economics and Business Administration.

Authors' Note: We are equal collaborators as editors and as authors and have listed our names in alphabetical order. 
Association for Business Communication. Most of the cases were researched in specific business situations and have been user-tested, often in classrooms at universities other than the author's own. All of the cases have been revised extensively on the basis of feedback from practitioners, instructors, and students. Summarized in the following list, the guidelines governing both the case selection and revision processes help to explain the characteristics of each case in this collection:

- Focuses on communication.

- Requires communications decision-making.

- Furnishes a business context.

- Presents an engaging story or scenario.

- Provides sufficient realistic detail for analysis and response.

- Functions readily in the classroom.

- Applies to a wide range of teaching philosophies and educational settings.

- Relates to contemporary issues and problems.

Each of these characteristics contributes to the usefulness of the cases for classroom instruction in business/management communication.

Cases offer many opportunities for communication instructors, opportunities which we explore in this introduction with examples drawn from the casebook. (See the appendix for guidelines.) The heart of the case method in business/management education, however, suggests the chief advantage of using cases in communication instruction-they provide a slice of business life inside the classroom. Cases are just that-instances of business experience. The case creates a realistic situation for all members of a class to experience the events in a company, enabling everyone to participate in both learning about and practicing business/management communication. A case provides the social dimensions for analyzing and discussing communication issues in context, and it furnishes the audiences a specific situation, a business setting, and a role for learners to perform as communicators in ways that more nearly represent writing and speaking in the workplace than most classroom-based pedagogies.

The multiplicity of examples in this casebook should offer cases relevant to the needs of many instructors of business and management communication. The casebook presents a provocative array of 
cases-a wide range of cases constructed in various sizes and shapes, out of different disciplinary assumptions, and for different pedagogical purposes and levels of learners. Cases range from brief scenarios of a few pages that could be analyzed in an hour or less (Pansedik case) to long, involved cases with many documents that could provide multiple assignments and absorb several class sessions of discussion and activities (L.A. Silks case). They represent researched cases constructed along the lines of conventional business cases, complete with detailed data about a real situation for learners to analyze and discuss (Denny's case) as well as simulated cases constructed as a trigger or prompt for a writing assignment (Rocky Mountain Mutual case).

Cases range from those more likely to be appropriate for undergraduates (Four Oaks Pavilion case) to those more likely to be suitable for MBA students (Statstar case).

Although there are marked contrasts among these cases on many dimensions, there are no categories assigned in this casebook. Indeed, we believe that cases should not be labeled as an MBA case, for example, because teachers, learners, and classroom situations vary widely; a case designed and used with MBA students might work very well for advanced undergraduates in some settings. Moreover, instructors can adjust assignments so that an MBA class might be charged with developing an overall communication strategy for a case whereas an undergraduate class might be assigned to prepare a single communication. The arrangement of the cases, therefore, eschews categories, inviting instructors to consider various options when selecting cases for their own classes.

Selecting cases from among those published in this casebook, incorporating them into courses, and using them with students should cause us to confront some of the critical issues about our pedagogy and its disciplinary bases. Traditionally, cases provide a link with business practices, helping instructors to make their teaching of business/management communication more relevant. But cases can also help define the discipline. Despite continuing efforts to develop business and management communication as an academic field, we also need to strive for a greater understanding of central theories and assumptions and how our pedagogy might both inform those constructs and how it might be guided by them. Our purpose here is to identify issues raised by case instruction, examining the challenges 
posed by teaching business/management communication in the classroom and exploring the opportunities cases afford to improve our instruction and contribute to our disciplinary conversation.

In this introduction, we discuss some of the instructional challenges and opportunities of teaching with cases in some detail. Presented in seven sections, the topics are interrelated, beginning with obvious advantages in using cases and moving to somewhat less apparent possibilities. Cases, we contend, offer the following opportunities:

- Provide the context for communicating.

- Enable active learning.

- Present communication as social action.

- Show the collaborative nature of communication.

- Integrate special topics like intercultural communication and ethics.

- Indicate communication effectiveness as contingent.

- Offer scenes to test communication theory and models.

In discussing these issues, we highlight various cases as examples.

\section{Cases Provide a Context for Communicating}

\section{Challenge}

Communication instruction in classrooms often tends to be detached from social context. Classroom activities frequently emphasize logical development of information and generic assignments addressed to an instructor who must evaluate rather than respond as a person. Although many instructors attempt to establish a community in the classroom so that learners communicate with real people who are involved with each other, this laudable effort to contextualize writing and speaking does not furnish a sufficient opportunity to explore workplace communication. Audiences composed of peer learners cannot, for example, begin to approximate the complexities of communicating with managers at several different levels of authority, together with peers and subordinates, as well as those outside the organization. Although the classroom context is a real one, classroom activities tend to be decontextualized from organizational pressures, and in this important respect they are radically different from activities in the workplace for which learners must be equipped to communicate. 


\section{Opportunity}

Cases provide a social world for communications-an audience of people with personalities, habits, and political alliances; a detailed, often messy situation fraught with business problems; and a context in an organization with some sense of place and a culture. Cases are stories, in effect, that tell what happened in a company from the perspective of someone within the firm, typically an entry- or middlelevel manager with whom a learner can identify (not the firm's executives). The stories reveal information in bits and pieces, much as the designated manager would have learned it on the job, and present much of it in informal conversational style. This slice of business life enables students to enter into the case world and to observe and participate in business communication in context, developing their own purposes and strategies for communicating.

The cases tell stories in which information gains meaning in relationship to the audiences, situations, and organizational contexts. In the Statstar case, learners recognize the imperative of analyzing their readers and considering the possible repercussions of identifying the boss himself as a cause of a client problem when preparing an e-mail for the manager in France. In the Four Oaks Pavilion case, learners recognize the importance of analyzing the political situation and carefully selecting their language (is it "noise" or "sounds of music" with which they are concerned?) when preparing a presentation for a public city council meeting.

Cases offer a social context for observing and/or performing communications in realistic situations when various organizational norms, political realities, and stakeholders with competing goals all come into play. Take, for example, the Langley case where the business problem revolves around issues of appropriate e-mail use and privacy. Here learners become members of a close-knit, highly effective work team who discover that their e-mail is being monitored by a manager whose unknown snooping finds the group's current social use inappropriate. As members of this group, learners must analyze a business problem conveyed largely through very informal conversation. They must collaboratively develop a group position, taking into account ethical and legal issues, as well as personal concerns that must be articulated in a professional environment. Defensiveness must give way to development of persuasive strategies for 
communicating with several players with different interests. The case then thrusts learners into a situation in which they must make communication decisions not only on the basis of ethics and employee morale but also on commonplace business realities.

Like the Langley case, most of the cases require learners to translate the case language into appropriate discourse for the assignments. Case language does not represent clear and concise business writing because a case is a fictional account designed for instructional purposes. Rather than presenting information efficiently, the case author attempts to convey the personalities and interaction of the characters as realistically as possible-using informal narrative and dialogue. Not only do these techniques make the case more engaging, but they enable learners to confront questions about what language and content is appropriate for communicating in different media, situations, and types of communications and for translating from one to another.

\section{Cases Enable Active Learning}

\section{Challenge}

Within the confines of the communication classroom, risks for poor performance are relatively limited compared with the workplace environment. Teaching students to accept the full responsibility for their communication decisions and to guide their actions accordingly is a difficult instructional task. Experiencing personal dissatisfaction from a low grade on a presentation or embarrassment at a less-thaneffective memo critiqued by a peer is a far cry from being advised that a proposal failed to make the final cut. Workplace writing and speaking carry a high level of responsibility, particularly given the increasingly litigious nature of business environments. Carelessness can incriminate. Imprudence can do injury. Intellectual understanding of how prudence must govern communication behavior can be facilitated by readings, discussions, and lectures, but experiential knowledge is far more powerful.

\section{opportunity}

Cases can move students from passive recipients of knowledge to active learners. While discussion of cases can demonstrate that communications have real consequences, it is the case itself that can pro- 
vide contexts in which learners begin to experience those consequences. In those cases that describe a negative situation-and ask "What went wrong here?"-learners are given an opportunity for retrospective analysis. This kind of analytical observation can become even more explicit when cases incorporate actual texts, such as the press release and internal memo in the Denny's case, the in-basket documents in the Sentry Assurance case, and the luncheon dialogue in the Pansedik case. Although identifying and learning from the mistakes of others goes some way toward illustrating the consequential nature of communications in the workplace, it does not go far enough.

Performance in response to case assignments is the chief way for learners to experience the consequences of communication. Traditionally, cases have been valued because they offer learners risk-free practice. If the assigned press release in the Four Oaks Pavilion case causes more citizen complaints, for example, the wrath of the city council members will not result in the writer's termination. But cases provide more than risk-free practice; they can also become occasions in which learners experience to some degree the weight of their communication decisions. Assignments for the cases in this volume require learners to accept specified roles and perform them within the context of the case. For example, the Stovall case asks respondents to draft a letter denying a customer claim. Not only does the letter have personal ramifications because the boss will evaluate it as part of a training program, but other managers might also review it, especially for possible legal ramifications.

Because each case depicts the audiences and the organizational community in some detail, peers can also assume audience perspectives and provide feedback as if they were actors in the case situation. Learners can try different strategies, actually playing out various responses and receiving feedback on them as if they were participants. For example, in the Rocky Mountain Mutual case, an executive named Zach Evans wants to close down the company's fitness center despite the pleas of those who are avid users, including the claims manager. In preparing their own communications, learners role-play an assistant to the claims manager, but later-because the case presents him in a fairly well-rounded picture-they can also role-play 
Zach Evans, the executive, and respond to memos trying to convince him to change his mind about the fitness center.

Granted, these experiences are simulated and the repercussions of failure are not felt as they would be in a real situation; but in the context of a case, learners can be held responsible for their words, particularly how those words might play out in the context of the particular business situation. Role-playing responses to such performances can allow learners to feel some sense of the ownership, responsibility, and scrutiny they can expect to experience in the workplace.

\section{Cases Present Communication as Social Action}

\section{Challenge}

Communication in classrooms tends to be treated as isolated, static products or events in specific formats. Instructors may teach modern concepts of communicative action or genres as social actions, but such ideas may be undermined by the ubiquitous assignment as a stand-alone response (give an informative presentation, write a badnews memo, prepare a feasibility study). Each assignment represents a particular format that the learner tries to complete, following whatever rules and models are available. Communication is not action in such a regimen of isolated assignments-not a way to accomplish tasks or to get work done. The isolated assignment also discourages learners from viewing communications as a larger strategy with a stream of multiple messages in multiple media. Instead, it is the single product, despite the fact that no one communication independently may be sufficient to solve a problem, persuade someone to change a plan, or negotiate a consensus between the two parties.

\section{Opportunity}

The stories presented in business and management communication cases include multiple interactions in mixed media and genres and show how these various types of communications are not empty forms but actions-ways to get things done-and how communication is a string or even a web of messages, not a single event. In the Statstar case, for example, the case story is told through a series of e-mail messages and letters with a client. The learner reads the messages, one by one, and must infer what is going on, gradually realizing that 
people are not communicating but miscommunicating-covering up, hiding, failing to take responsibility for their communicative actions.

In some cases, many documents representing several genres can be examined in the case. Rather than viewing samples or models of a genre, the learner reads these documents in context and thereby can more readily perceive the communicative action. This is, of course, the way new employees learn about new genres in the workplace, encountering actual documents as they play their role in unfurling events. The Denny's case, for instance, includes actual communications-a press release, an open letter to customers and communities, an internal memo-that were instrumental in that company's attempts to recover from charges of discrimination.

In addition, cases provide impetus for learners' development of various genres to accomplish tasks, including devising communication strategies for dealing with a situation; selecting specific media and genres; and determining the purpose for each communication. The American Electrical case, for example, closes with an environmental crisis on its hands. Management must communicate the company's position almost immediately to all the company's stakeholders. Although for the instructor's convenience the assignments for the American Electrical case (and all the other cases in this collection) suggest specific documents, the instructor can choose to leave the task open-ended, requiring learners to engage in developing and executing the full communication strategy, including media and genre selection.

In other cases, a string of communicative actions is at the core of the problem. The Information Solutions case, for example, presents a situation in which diverse internal groups neither understand nor effectively interact with each other; hence business suffers. The Sentry Assurance case involves a buy-out by which the once effective lines of internal communications in an established firm become challenged, even threatened, by the infusion of new employees from the buy-outnew employees who have very different modes of operating and communicating. The lack of written communications and the need to obtain those documents quickly through communications across time zones and, even more significant, across cultures represents the problem in the case aptly titled Lester Crane: Getting Approvals After the Fact. 


\section{Cases Show the Collaborative Nature of Communication}

\section{Challenge}

Teaching communication as inherently collaborative is difficult in the classroom. Business/management communication courses frequently include a variety of collaborative activities - team projects, presentations, and reports--all of which introduce learners to the benefits and pitfalls of collaborative work. Such activities also provide an occasion for instructors to introduce principles for more effective work negotiation, group presentation, and collaborative writing. These activities themselves, however, tend to be completed through division of labor rather than through full collaboration.

Another shortfall arises because typical collaborative classroom activities may not go far enough for learners to recognize that workplace communication is a collaborative, not an individual, enterprise. Granted, activities such as peer editing, including document exchange and review, can help learners begin to understand the very public nature of communications in professional environments-e.g., their writing is likely to be critiqued by others. But such activities are only partially successful in helping learners understand what it means to write on behalf of another, be it an individual, a group, or an organization. Yet collaborative ownership of communications, be they written or oral, is at the core of organizational life and business practice.

\section{Opportunity}

The stories that cases tell not only show collaboration at work but also can engage learners in collaborative discourse simulating real business situations. On one level, the common context provided by the case becomes a shared basis for collaborative problem-solving reflective of the workplace. On another level, case assignments ask learners to communicate as participants inside the case scene, enabling them to experience what collaboration may mean in the workplace. For example, the Stovall case involves users in collaborative analysis and drafting of documents; the Davis-Martin case requires difficult negotiation and compromise in a situation where personal beliefs conflict with organizational goals; and the Information Solutions case involves learners in a task force that must devise a plan to improve communications among diverse groups currently unable to work together. In sum, case assignments can provide a wide 
range of collaborative experiences unavailable in traditional writing and speaking exercises.

\section{Cases Integrate Special Topics Like Interculturalism, Ethics}

\section{Challenge}

Although it is increasingly recognized that special topics internationalism, interculturalism, ethics, gender, and electronic media highly impact communication and should be incorporated into communications courses, finding time in already crowded curricula is not easy. Even more problematic than timing and placement, however, is the issue of integration: How does a communication instructor get beyond the topical unit approach (e.g. "Emerging Ethical Considerations" or "Managing Communication Technology") and provide classroom experiences that allow learners to absorb the intimate connections between their communications and, say, their gender, values, and cultural identity? How can these topics be personalized and contextualized so that learners begin to see the complex and consequential realities involved?

\section{Opportunity}

Cases immerse learners in situations where special topics are contextual realities woven into the very fabric of the story. These special topics become social issues that are intertwined with each other and intimately connected to communication. Sometimes a particular issue may be highlighted in a case, as, for example, international and intercultural issues dominate the L. A. Silks case which describes a Los Angeles firm's failed attempt to expeditiously negotiate entrance of business into China. The American and Chinese businesspersons in the L.A. Silks case often miscommunicate because of their radically different assumptions about the purpose for their correspondence and how it should go forward. In effect, ignorance of cultural differences gives rise to mistrust on both sides, as each correspondent fails to understand or meet the expectations of the other.

International issues also are significant in the Lester Crane case (which involves product shipments from the US to Saudi Arabia), the TeknoSport case (which concerns a sports training equipment firm located in Finland), and the Information Solutions case (which occurs in New Zealand). Legal issues play an important role in the Stovall and 
the Denny's cases. Environmental concerns are foremost in the Four Oaks Pavilion and the American Electrical cases. Ethical dilemmas concern the employees in both the Davis-Martin and Trustworthy Insurance cases. Electronic media affect many cases, but are central in the Information Solutions, Langley, and Statstar cases.

Yet, none of these cases revolves around a single issue. Cases by nature present an intermingling of issues, none of which is isolated from the other and none of which is simple. The blend of various topics that are of particular interest for business/management courses is aptly illustrated by the cases in this collection. The Sentry Assurance case narrates a situation where it initially appears that a salesperson has stolen the client of a colleague-clearly an unethical act. Yet, further analysis suggests a cold business reality, namely that the client, who is a man, seems to have preferred to deal with the male salesperson and simply exercised his privilege as the customer to do so. Suddenly, a supposedly straightforward ethical matter becomes far more complicated. Such intrusions of gender issues occur in other cases as well, including the Davis-Martin case and, most blatantly, in the Lester Crane case, where the American manufacturer typically keeps its women managers out of the Middle East.

As these examples suggest, cases lend themselves to dealing with a variety of issues simultaneously. Cases dramatize and personalize the interrelationships among issues. On a very practical level, cases remove the necessity of having to designate segments of a course to various topics, some of which the instructor may not feel entirely comfortable teaching anyway. Because these topics naturally emerge from case discussions, they will become part of the curriculum without any formal designation. But more importantly from an instructional perspective, cases allow such topics to arise inductively. Engaging with the case, individuals are more likely to share their experiences and to discover their own attitudes and tendencies. Also, case assignments cause learners to struggle with topics that have a long-term impact on their communication strategies and choices-for example, learning about cultural differences can help respondents to the L.A. Silks case prepare communications for the Chinese contacts, but becoming aware of their own and others' intercultural ignorance may be more significant. 


\section{Cases Show Communication Effectiveness as Contingent}

\section{Challenge}

The notion that communication training supplies right answers and stock solutions persists. Despite long-time efforts to debunk them, formulaic approaches (use active verbs, insert a buffer, provide reader benefits) continue to find an audience, and businesses continue to hire trainers to teach half-day workshops to correct employees' communication deficiencies. In this context, learners expect to be provided with rules to follow, making it difficult to convince them that there is no right way in any situation but, rather, any number of alternatives that may be functionally effective. Communication effectiveness differs from one context to the next, as well as one community and one individual to the next.

\section{Opportunity}

Cases confront users with workplace problems for which there are no right answers. At the conclusion of the classic business case is this question: "What would you do now if you were in this situation?" While some may assume that there is a best response-perhaps the way the actual company responded, the solution favored by the instructor, or one a consultant would devise-this is not true. In fact, researched cases are predicated on the assumption that what happened after the case ends is irrelevant (though highly interesting, of course). Rather, the case is a teaching method that poses problems for the learners to confront and fashion solutions-either for defending during class discussion or for executing in oral or written performances. The notion is to develop and compare different strategies for solving problems, strategies that may be equally in effective or effective to varying degrees.

Comparing diverse responses to cases becomes a way to extend the concept of evaluation as contextually dependent and subjective. Evaluations of workplace communication effectiveness depend on the context-the set of circumstances at a particular moment, the organizational culture, the discourse community, and the speakers/authors. One theoretical stance toward this dependency on context suggests that effectiveness is determined by comparing what a particular message achieves with what it needs to achieve for the given situation 
(Quinn, Hildebrandt, Rogers, \& Thompson, 1991). Another has been characterized as the "discrepancy between receiver expectations about and perceptions of technical, functional, and aesthetic communication processes and outcomes" (Shelby, 1994, p. 11), an idea recently elaborated as "fitness for use" (Shelby, 1997). In short, communications that are effective in one instance may fail in another.

Furthermore, the effectiveness of communications depends on their suitability for the ethos of the speaker and/or writer. Not only does the choice of authors or speakers influence the evaluation of a communication, but the communication must represent the individual as well as convey an individual perspective. In fashioning responses, learners should draw inferences from the information presented in the case, building up a framework inductively from the data as well as from individual knowledge and experience. (Rangan, 1996) Out of this effort, each learner creates his or her own perspective, a principle that is reinforced throughout the assignments in this casebook. Inferencing is essential to create a real understanding of any case situation. Learners should not confine themselves to the surface facts of a case, but neither should they fabricate information. Respondents to the Rocky Mountain Mutual case, for example, may apply what they know about the benefits of exercise to argue for the maintenance of a company health club. Bringing their own ideas to bear upon the problems in the case, respondents learn to devise answers that are uniquely their own yet operable within the constraints of the case situation. Cases then suggest the kind of individual contribution that is expected of professionals in the workplace and the key role that communication plays in their eventual ability to make that contribution.

In contrast to business cases that ask learners to assume the enlarged perspective of executive level management, most communications cases ask what the individual holding an entry-or mid-level position should do now. Fashioning a response then means presenting one's individual ethos as a potential manager-determining how to sound in this situation, what is a comfortable persona to assume that coincides with the individual's management style, personality, approach to business life (Statstar case). In some case assignments, learners must communicate on behalf of another; in such an instance, individual ethos is subordinated in developing an appropri- 
ate voice for the manager who will "author" (that is sign) the communication, a common workplace arrangement (Four Oaks Pavilion case, L.A. Silks case). In other case assignments, individual authors or speakers represent the company more than themselves as individuals (American Electrical case). In still other cases, the student role-plays a designated individual who holds a particular position in an organizational community (such as Joe Terrell in Sentry Assurance, Mary Anderson in Pansedik, and Rae Severns in Davis-Martin).

\section{Cases Offer Scenes for Testing Theories and Models}

\section{Challenge}

Business/management communication is a relatively new field with unevenly developed theories and principles, few of which enjoy disciplinary consensus. "Business communication research," wrote Smeltzer and Suchan in a Journal of Business Communication editorial, "continues to represent a pastiche of theoretical perspectives borrowed from organizational behavior, speech communication, rhetoric, composition, organizational communication, marketing, international business, and a number of other areas" (1991, p.181). The same could be said of our pedagogy. Efforts to characterize standard course content have included a variety of program surveys and analyses of syllabi (Bogert \& Butt, 1996; Munter, 1995; Stanford University, 1997), all of which suggest that there is little consensus on the essential theories and principles we should teach. Meanwhile, journal articles claim disciplinary associations, debate and test the relevance of various theories, and originate theoretical constructs for business and management communication (e.g., Forman, 1993; Shelby, 1997). As we build theory, we need to test and apply it not only in the workplace but in our pedagogy, recursively refining it and attempting to create consensus on what constitutes knowledge in this field.

\section{opportunity}

Cases provide a unique opportunity to explore the applicability of theoretical constructs, frameworks, and models. To the extent that cases mirror real and often messy situations that defy simplistic interpretations and responses, they invite the application of theories, or sense-making frameworks, and do so in a way that narrow pedagogical exercises cannot. In fact, some constructs are highly relevant to 
particular cases. The Trustworthy case, for example, asks users to determine when and how to tell employees that their company is moving-decisions that can be facilitated by considering the power bases and verbal strategies associated with compliance-gaining theory, particularly as it is reinterpreted by David and Baker (1994). In response to the Trustworthy case, compliance-gaining theory can be used to argue that employee acceptance may more readily be obtained if the announcement of the move comes from the CEO who has legitimate and expert power by the nature of his position and if it is articulated using verbal strategies reflective of that power ("I know that this move will make it more difficult for some of you to get to work, but the move is vital if the company is to grow and remain competitive.").

Cases also have a way of revealing the limitations of any particular theory and the value of applying more than one construct to a particular case. Also, for example, in the Trustworthy case the need for a faceto-face meeting between the CEO and employees seems validated by media richness theory (Trevino, Lengel, \& Daft, 1990), which posits that written media are less rich and provide too few cues and thereby should not be used for non-routine communications (as is the situation here). Yet, media richness does not account for the potential richness that can reside in a CEO's written statement, richness that may come through a formal declaration of how and why the move will happen. In such instances, a written document constitutes a contract or agreement between parties that-at least in some cultures-is much more tangible than a handshake. The point is that theory can assist learners in making sense of a complex situation in a case, at least to some degree, but cases also challenge and test the limits of theory.

\section{Conclusion}

We hope that this casebook will encourage many instructors who have never taught by the case method to try it out. We hope that experienced case instructors will feel nudged to add new cases to their repertoire, cycling out some of the older models. And we also hope that instructors will discuss cases and case teaching experiences within our academic community, including the sharing of sample 
responses to cases and ideas about how to adapt the cases provided here to their own situations on $B C Q$ on the Web.

Using a common set of cases and sharing our experiences might generate communal engagement with the case method, eventually sparking debate and inquiry that could make case research into a legitimate, scholarly component in business/management communication. In most business disciplines and in allied fields like communication, case pedagogy and research are very important parts of disciplinary activity (for example, see Keough, forthcoming). Perhaps the time is near when we will make it part of our discipline as well.

\section{References}

Bogert, J., \& Butt, D. (1996). Communication instruction in MBA programs: A survey of syllabi. Business Communication Quarterly, 59(2), 20-44.

David, C., \& Baker, M. S. (1994). Rereading bad news: Compliance-gaining features in management memos. Joumal of Business Communication, 31, 267-290.

Forman, J. (1993). Business communication and composition: The writing connection and beyond. Journal of Business Communication, 30, 333-351.

Keough, C. (forthcoming). The case of the aggrieved expatriate. Management Comnunication Quarterly.

Munter, M. (Ed.) (1995). Management communication: Business administration reading lists and course outlines. Chapel Hill, NC: Eno River Press.

Quinn, R. E., Hildebrandt, H. W., Rogers, P. S., \& Thompson, M. P. (1991) A competing values framework for analyzing presentation communication in management contexts. Joumal of Business Communication, 28, 213-232.

Rangan, V. K. (1995). Choreographing a case class. (Case No. 595-074). Boston: Harvard Business School Publishing.

Shelby, A. N. (1997). Quality communication revisited: Exploring the link with persuasive effects. Outstanding Researcher Award Lecture, Association for Business Communication Conference, Washington DC.

Shelby, A. N. (1994). Communication quality as a metacommunication: A conceptual analysis. In L. van Waes, E. Woudstra, \& P. van den Hoven (Eds.), Functional communication quality: Utrecht studies in language and communication (pp. 5-16). Amsterdam: Rodopi.

Smeltzer, L. R., \& Suchan, J. E. (1991). Guest editorial: Theory building and relevance. Joumal of Business Communication, 28, 181-186.

Stanford Benchmarking Survey (Spring/Summer, 1997). MBA management communication. Stanford University Graduate School of Business, Stanford, CA.

Trevino, L., Lengel, R., \& Daft, R. (1990). Understanding manager's media choices: A symbolic interactionist perspective. In J. Fulk \& C. Steinfield (Eds.), Organizations and Communication Technology (pp. 71-94). Newbury Park, CA: Sage. 


\section{Appendix}

\section{Practical Tips for Assigning Cases}

Below are some practical tips for assigning cases and assisting students so that they might get more value out of the case experience in business/management communication courses.

\section{Discuss Role Assignments}

Advise respondents to note who they represent in writing or speaking assignments. If they are asked to write on behalf of someone else, they should assume that role. Otherwise, they should be themselves as if they were employees of the organization in the case. Most case assignments call for performers to play the part of an employee holding a specific position in the depicted company, but not role-play an individual person with a name, gender, age, personality, values. Those who communicate in the designated position in the firm must accept the inherent constraints of that job, but each should imagine him or herself to be in the position, not be some other person. Rather than pretending to be someone else, learners should try to imagine themselves acting in the designated job, behaving and sounding as appropriate for their individual professional selves.

Suggest learners write their own names on the top page of case responses to avoid problems in identifying authors. Since many cases ask learners to draft for a supervisor, every writer will have the same supervisor's name on the "from" line of some case documents.

\section{Guide Performance and Language Use}

Discuss rules for playing the case game in your classroom. The instructions throughout this casebook recommend drawing inferences from case information, as well as the learners' own experience and knowledge, but prohibit inventing information. If you expect learners to follow these guidelines, explain them fully. Also, if you expect learners to research issues raised in a case, announce that task when you assign the case.

Discuss the language of the case so that learners recognize that the informal oral style may require transformation into more formal oral or written discourse. Word choices, syntax, grammar, and even matters such as what to say are governed by context, medium, and genre, as well as audience, situation, and community norms. Most information in cases cannot be directly transferred into documents or oral performances in response to the case.

\section{Attach Specific Dates}

Provide instructions on the years to be used in formulating communications from each case. Dates furnished in cases omit years to enable instructors to make actions appear current; writers should not represent dates in their documents in this fashion (e.g. , June 13,0000). Indicating a 
specific year will enable dates for case information to be provided as well. Although the zeros used to represent years should be transformed when using a case, inserting zeros for zip codes and area codes merely avoids any possibility of publishing real addresses or telephone numbers. Tell learners to ignore them.

\section{Develop Your Own Questions}

Consider developing questions that will focus vour class on specific issues in the case that will fit with your learning objectives. Because most cases are suitable for applying a wide range of theories and approaches, your class may benefit more by specific questions which reflect your goals rather than a general set of study questions. Consult $B C Q$ on the Web for suggested study questions and other teaching notes on most cases.

\section{Select Assignments}

Rather than distributing all the assignments (some of which may be options), create your own assignment sheet before photocopying the case for learners. Select any assignments you plan to use from the separate section at the end of the case, cutting and pasting to produce your own assignment sheet, or adapt one or more assignments to your circumstances to create a custom-made set of assignments. Another alternative is to assign learners to develop their own tasks, determining what communications media and genres would best accomplish their goals. If the case has intermediate assignments integrated into the narrative, make it explicit whether you expect these tasks to be completed. (If the case has intermediate tasks or divides easily into sections, you might prefer to distribute it in pieces over time so that classes could discuss issues or do assignments before learning of later developments.) 